\title{
Holonomy and Equivalence of Analytic Foliations *
}

\author{
Francisco Chaves
}

April 13, 2021

\begin{abstract}
The main goal of this paper is the analytic classification of the germs of singular foliations generated, up to an analytic change of coordinates, by the germs of vector fields of form the $x \partial_{x}+\sum_{i=1}^{n} a_{i}(x, \mathbf{z}) \partial_{z_{i}}$, where $a_{i}(x, \mathbf{z})$ is a germ of analytic function with $a_{i}(x, 0)=0$. We prove, under some hypothesis, that these germs of singular foliations are analytically classified once their local holonomy along a given separatrix are analytically conjugated.
\end{abstract}

\section{Introduction}

The analytic classification of singular analytic foliations in dimension two and its connection with the analytic conjugation of the corresponding holonomies was one of the central results of the well-known paper of Mattei and Moussu MM80 in 1980.

Later in 1984, Elizarov and Il'Yashenko [EI84 proved that, in dimension three, if we add some restrictions on the vector field that generates the foliations, the analytic conjugation of the holonomies corresponds to the analytic equivalence of the foliations. In the year 2006, Helena Reis Rei06 reproved a result of the same type, but for higher dimensions.

In more details, the authors consider germs of singular analytic vector fields $X$ in $\left(\mathbb{C}^{n}, 0\right)$ for $n \geq 3$, with $\lambda_{1}, \ldots, \lambda_{n}$ as the eigenvalues of the linear part of $X$, verifying:

1. The origin of $\mathbb{C}^{n}$ is an isolated singularity of $X$.

2. $X$ is of Siegel type (i.e, the convex hull of the eigenvalues of its linear part contains the origin).

*I am deeply grateful to my thesis advisor Prof. D. Panazzolo who proposed this problem and made crucial contributions in many results in this paper. Without his participation, this paper would not be done. Also, I thank Prof R. Targino for the suggestions to improve the quality of the text. The author is glad to be supported by the Université de Haute-Alsace (UHA), where my Phd training has been well assisted by the IRIMAS laboratory. 
3. All the eigenvalues of the linear part of $X$ are nonzero and there exists a straight line through the origin of $\mathbb{C}$ separating $\lambda_{1}$ from the others eigenvalues in the complex plane.

4. Up to a change of coordinates, $X=\sum_{i=1}^{n} \lambda_{i} z_{i}\left(1+f_{i}(z)\right) \partial_{z_{i}}$, where $z=$ $\left(z_{1}, \ldots, z_{n}\right)$, and $f_{i}$ is a germ of analytic function such that $f_{i}(0)=0$ for all $i$.

In [EI84] and [Rei06], it is proved the following.

Proposition ([Rei06], Theorem 1). Let $X$ and $Y$ be two germ vector fields, verifying (1), (2), (3) and (4). Denote by $h_{X}$ and $h_{Y}$ the holonomies of $X$ and $Y$ relatively to the separatrices of $X$ and $Y$ tangent to the eigenspace associated with the first eigenvalue, respectively. Then, if $h_{X}$ and $h_{Y}$ are analytically conjugated, $X$ and $Y$ are analytically equivalent.

In this paper, we drop the hypothesis (1), and weaken (2), (3), and (4). As consequence, we enlarge the set of vector fields for which the conclusion of the theorem holds.

More precisely, we treat the class of germs of singular analytic foliations called crossing type. A crossing type foliation in $\left(\mathbb{C}^{n+1}, 0\right)$ is a triple $(\mathcal{F}, H, \Gamma)$ such that:

i. $\mathcal{F}$ is a germ of 1-dimensional analytic foliation.

ii. $H$ is a smooth hyper-surface and $\Gamma$ is a smooth invariant curve such that:

(a) $H$ and $\Gamma$ are transverse at the origin.

(b) Both are invariant by the foliation $\mathcal{F}$.

iii. Each local generator of $\mathcal{F}$ has a nonzero eigenvalue in the $\Gamma$-direction.

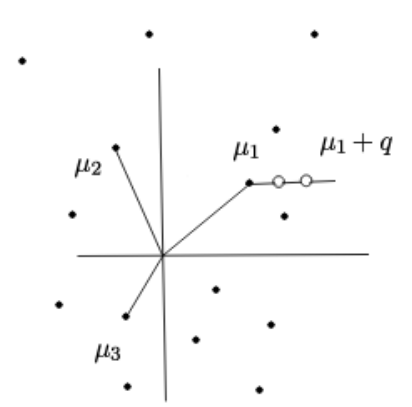

Figure 1: Transversality of $H$ and $\Gamma$ at origin

As in the papers cited above, we have to demand a property on the eigenvalues of the linear part of the local generators. We say that a vector field, 
with $1, \mu_{1} \ldots, \mu_{n}$ as the eigenvalues of its linear part, has no transverse negative resonance if no element in the positive cone $\mathcal{C}=\left\{\sum_{i=1}^{n} p_{i} \mu_{i} ; p_{1}+\cdots+p_{n} \geq 1\right\}$, where $p_{i} \in \mathbb{Z}_{\geq 0}$, can be written in the form $\mu_{j}+q$, with $q \in \mathbb{Z}_{\geq 1}$, for any $1 \leq j \leq n$.

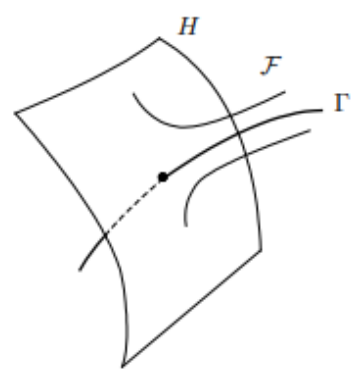

Figure 2: No transverse negative resonance

As consequence of the definition of crossing type foliation, there exist local coordinates $(x, \mathbf{z})$, so-called adapted to $(\mathcal{F}, H, \Gamma)$, such that the curve $\Gamma$ and the hypersurface $H$ are expressed respectively by $\Gamma:=\{\mathbf{z}=0\}, H:=\{x=0\}$. Moreover, if the local generators of $(\mathcal{F}, H, \Gamma)$ have no transverse negative resonance, we can choose a local generator in these adapted coordinates which has the form

$$
x \partial_{x}+\sum_{i=1}^{n} \sum_{j=1}^{n} a_{i j} z_{j} \partial_{z_{i}}+\sum_{i=1}^{n} b_{i}(x, \mathbf{z}) \partial_{z_{i}}
$$

where $\left(a_{i j}\right)_{n \times n}$ is a constant matrix, and $b_{i}(x, 0)=\frac{\partial b_{i}}{\partial z_{j}}(x, 0)=0$ for all $i, j \in$ $\{1, \ldots, n\}$. We say that a vector field of this form is an $x$-normalized vector field.

Our main goal is to classify such singular foliations up to analytic equivalence. Here, we say that two crossing type foliations $(\mathcal{F}, H, \Gamma)$ and $(\mathcal{G}, L, \Omega)$ are analytically equivalent if there exists an analytic change of coordinates mapping the leaves of $\mathcal{F}$ to the leaves of $\mathcal{G}$ and the pair $(H, \Gamma)$ to $(L, \Omega)$.

The following theorem is the main result in this paper. We adapted and generalized an idea originally introduced in the thesis of Arame Diaw [Dia19, DL20] to prove it. Below, we denote by $\Gamma$-holonomy and $\Omega$-holonomy the respective local holonomies along the curves $\Gamma$ of $\mathcal{F}$ and $\Omega$ of $\mathcal{G}$.

Main Theorem. Let $(\mathcal{F}, H, \Gamma)$ and $(\mathcal{G}, L, \Omega)$ be two crossing type foliations such that:

(a) The linear part of the local generators of $\mathcal{F}$ and $\mathcal{G}$ are conjugated.

(6) The local generators of $\mathcal{F}$ (and therefore that of $\mathcal{G}$ ) have no transverse negative resonance.

(c) The respective $\Gamma$-holonomy and $\Omega$-holonomy are analytically conjugated. 
Then, $(\mathcal{F}, H, \Gamma)$ and $(\mathcal{G}, L, \Omega)$ are analytically equivalent.

Motivated by the Main Theorem, we say that a crossing type foliation $(\mathcal{F}, H, \Gamma)$ is analytically classified by its linear part and its $\Gamma$-holonomy if all crossing type foliations $(\mathcal{G}, L, \Omega)$ with a conjugated linear part and a conjugated $\Omega$-holonomy to $(\mathcal{F}, H, \Gamma)$ is analytically equivalent to $(\mathcal{F}, H, \Gamma)$.

As a consequence of this theorem, we can give a unified proof for a result obtained with different tools by Mattei and Moussu MM80 and later by Martinet and Ramis MR82. In a recent paper DL20, Diaw and Loray also use similar techniques to reprove this theorem. In our notation, we can enunciate it as follows.

Corollary 1. Consider a crossing type foliation $(\mathcal{F}, H, \Gamma) \in\left(\mathbb{C}^{2}, 0\right)$ which has, in adapted coordinates, a x-normalized local generator of the form

$$
X=x \partial_{x}+y f(x, y) \partial_{y},
$$

where $f$ is a germ analytic function such that $f(0,0)=\lambda$. Then, two cases can occur.

1. The eigenvalue $\lambda$ belongs to $\mathbb{C} \backslash \mathbb{R}_{\leq 0}$, then $(\mathcal{F}, H, \Gamma)$ is analytically linearizable.

2. The eigenvalue $\lambda$ belongs to $\mathbb{R}_{\leq 0}$, then $(\mathcal{F}, H, \Gamma)$ is analytically classified by its linear part and its $\Gamma$-holonomy.

We recall that a germ of singular vector field in $\left(\mathbb{C}^{n}, 0\right)$ with eigenvalues $\lambda_{1}, \ldots, \lambda_{n}$ is in the Siegel (resp. Poincaré) domain if the origin lies (resp. does not lie) in the convex hull of the eigenvalues in $\mathbb{C}$.

Proof. In the Poincaré case $\left(\lambda \in \mathbb{C} \backslash \mathbb{R}_{\leq 0}\right)$, the result is immediate since the existence of two analytic separatrices implies that there can be no resonance of Poincaré type. In the Siegel case $\left(\lambda \in \mathbb{R}_{\leq 0}\right)$, it is sufficient to remark that the condition of no transverse negative resonance is satisfied. Hence, the result is a consequence of the Main Theorem.

In dimension three, a generalization of this result, which will be proved in section 3 , is the following corollary.

Corollary 2. Consider a crossing type foliation $(\mathcal{F}, H, \Gamma) \in\left(\mathbb{C}^{3}, 0\right)$ which has, in adapted coordinates, a x-normalized local generator with semi-simple part

$$
x \partial_{x}+\lambda y \partial_{y}+\mu z \partial_{z} .
$$

Then, three cases can appear:

1. The eigenvalues are in the Poincaré domain. Then $(\mathcal{F}, H, \Gamma)$ is analytically normalizable and has at most a finite number of resonant monomials. 
2. The eigenvalues are in the Siegel domain and at least one of the eigenvalues $\lambda, \mu$ is non-real. Then $(\mathcal{F}, H, \Gamma)$ is analytically classified by its linear part and its $\Gamma$-holonomy.

3. The eigenvalues are in the Siegel domain and all real. Then, either $(\mathcal{F}, H, \Gamma)$ is analytically classified by its linear part and its holonomy or one of the following conditions holds up to a permutation of the $y$ and $z$ coordinates:

(a) Either $\mu<\lambda \leq 0$ and

$$
p \lambda=\mu+q,
$$

for some $p, q \in \mathbb{Z}_{\geq 1}$.

(b) Or, $\mu \leq 0<\lambda$, and either (2) holds or $\lambda \in \mathbb{Q}_{>0}-\mu \mathbb{Q}_{\geq 0}$ (notice that these conditions are not mutually exclusive).

The basic tool used in this paper is the concept of $D_{r, R^{-} \text {transversely for- }}$ mal series. A $D_{r, R}$-transversely formal series is a formal series of the form $\sum_{k_{i} \in \mathbb{N}} f_{K}(x) \mathbf{z}^{K}$, where $\mathbf{z}^{K}=z^{k_{1}} \ldots z^{k_{n}}$, and each coefficient $f_{K}(x)$ is convergent in the annulus $D_{r, R}:=\{x \in \mathbb{C} ; r<|x|<R\}$, where $r, R>0$.

The $D_{r, R}$-transversely formal derivations (derivations over the ring of the $D_{r, R}$-transversely formal series) can be seen as vector fields with coefficients being $D_{r, R}$-transversely formal series. We study the exponential map, the normal form, and the symmetries for such derivations.

After a general study of these objects, we focus on a specific class of vector field. We say that a $D_{r, R}$-transversely formal vector field is $x$-normalized if it has the form (1), and $b_{1}, \ldots, b_{n}$ all lie in the ideal generated by monomials of the form $z_{i} z_{j}$ for $i, j \in\{1, \ldots, n\}$ in the ring of $D_{r, R}$-transversely formal series.

If the components $f_{K}$ of a $D_{r, R}$-transversely formal vector field converge in the disk $D_{R}:=\{z \in \mathbb{C} ;|z|<R\}$, where $R>0$, we say that the vector field is a $D_{R}$-transversely formal vector field. Connecting these two classes of vector fields, the following result is a central step to the proof of the Main Theorem.

Theorem 3. Let $X$ be an $x$-normalized $D_{R}$-transversely formal vector field that has no transverse negative resonance and $Y$ be an x-normalized $D_{r, R^{-}}$ transversely formal vector field. If $[X, Y]=0$, then $Y$ is an $x$-normalized $D_{R}$-transversely formal vector field.

\section{Transversely Formally Objects.}

\subsection{The Ring $\mathcal{O}_{r, R}[[\mathrm{z}]]$.}

The basic assumptions and notations for this paper are:

1. The set of natural numbers $\mathbb{N}$ contains zero.

2. The set $\left\{e_{1}, \ldots, e_{n}\right\}$ is the canonical basis of $\mathbb{R}^{n}$.

3. $\mathcal{L}_{n}$ is the set of $n$-uples $K \in\left\{\left(\mathbb{N}^{n}-e_{1}\right) \cup \cdots \cup\left(\mathbb{N}^{n}-e_{n}\right)\right\}$, such that $|K|=k_{1}+\cdots+k_{n} \geq 0$. 
4. $\mathcal{L}_{n, m}$ is the set of $n$-uples $K \in \mathcal{L}_{n}$, such that $|K|=k_{1}+\cdots+k_{n} \geq m$.

5. The $\sup _{A} f$ denotes the supremum of the function $f$ on the set $A$.

6. We denote respectively by $\mathcal{O}_{r, R}$ and $\mathcal{O}_{R}$ the rings of germs of analytic functions on $D_{r, R}$ and $D_{R}$.

7. A point $p \in \mathbb{C}^{n+1}$ is denoted by $p=(x, \mathbf{z})=\left(x, z_{1}, \ldots, z_{n}\right)$.

We now recall the construction of the ring $\mathcal{O}_{r, R}[[\mathbf{z}]]$ of formal power series of $n$ indeterminates $z_{1}, \ldots, z_{n}$ with coefficients in $\mathcal{O}_{r, R}$. For it, we are going to use the notion of inverse limit.

Consider the ideal $\mathfrak{m}:=\left\langle z_{1}, \ldots, z_{n}\right\rangle \subset \mathcal{O}_{r, R}[\mathbf{z}]$. Let $\left(J^{(i)},\left(\pi_{j i}\right)\right)_{j \leq i \in \mathbb{N}}$ be the inverse system indexed by $\mathbb{N}$, where $J^{(i)}=\mathcal{O}_{r, R}[\mathbf{z}] / \mathfrak{m}^{i+1}$, and for each $j \leq i$, $\pi_{j i}: J^{(i)} \rightarrow J^{(j)}$ is the linear map with kernel $\mathfrak{m}^{j+1}$ called bonding map. We define

$$
\mathcal{O}_{r, R}[[\mathbf{z}]]:=\lim _{\coprod_{j \in \mathbb{N}}} J^{(i)}=\left\{f \in \prod_{i \in \mathbb{N}} J^{(i)} ; f_{j}=\pi_{j i}\left(f_{i}\right) \text { for all } i \leq j \in \mathbb{N}\right\} .
$$

An element $f \in \mathcal{O}_{r, R}[[\mathbf{z}]]$ is called a $D_{r, R}$-transversely formal series, and we can write $f(x, \mathbf{z})=\sum_{|K|=0}^{\infty} f_{K}(x) \mathbf{z}^{K}$, where $\mathbf{z}^{K}=z^{k_{1}} \ldots z^{k_{n}},|K|=k_{1}+\cdots+k_{n}$, and each coefficient $f_{K}(x)$ is convergent in the annulus $D_{r, R}$. We still denote by $\mathfrak{m}$ the maximal ideal $\left\langle z_{1}, \ldots, z_{n}\right\rangle \subset \mathcal{O}_{r, R}[[\mathbf{z}]]$.

Definition 1. We say that $f \in \mathcal{O}_{r, R}[[\mathbf{z}]]$ is convergent if for each $r^{\prime}, R^{\prime}$ with $0<r<r^{\prime}<R^{\prime}<R$, there exist constants $C, M \in \mathbb{R}$ such that $\sup _{D_{r^{\prime}, R^{\prime}}}\left\|f_{K}\right\| \leq$ $C M^{|K|}$. We denote by $\mathcal{O}_{r, R}\{\mathbf{z}\} \subset \mathcal{O}_{r, R}[[\mathbf{z}]]$ the ring of $D_{r, R}$-transversely convergent series.

We can define exactly in the same way as $\mathcal{O}_{r, R}[[\mathbf{z}]]$ the subring $\mathcal{O}_{R}[[\mathbf{z}]] \subset$ $\mathcal{O}_{r, R}[[\mathbf{z}]]$ of formal power series of $n$ indeterminates with coefficients in $\mathcal{O}_{R}$. An element $f \in \mathcal{O}_{R}[[\mathbf{z}]]$ is called a $D_{R}$-transversely formal series.

Definition 2. We say that $f \in \mathcal{O}_{R}[[\mathbf{z}]]$ is convergent if for each $R^{\prime}$ with $0<$ $R^{\prime}<R$, there exist constants $C, M$ such that $\sup _{D_{R^{\prime}}}\left\|f_{K}\right\| \leq C M^{K}$. We denote by $\mathcal{O}_{R}\{\mathbf{z}\} \subset \mathcal{O}_{R}[[\mathbf{z}]]$ the ring of $D_{R}$-transversely convergent series.

We observe that diagram of inclusions below follows directly from the definitions.

In the next lemma, we establish that the ring in the last row is the intersection of the two in the middle line.

Lemma 4. $\mathcal{O}_{r, R}\{\mathbf{z}\} \cap \mathcal{O}_{R}[[\mathbf{z}]]=\mathcal{O}_{R}\{\mathbf{z}\}$.

The proof is based in the next claim.

Claim 1. Let $f$ be an analytic function on $D_{R}$. Then $\sup _{D_{r, R}}\|f\|=\sup _{D_{R}}\|f\|$, for any $0<r<R$. 


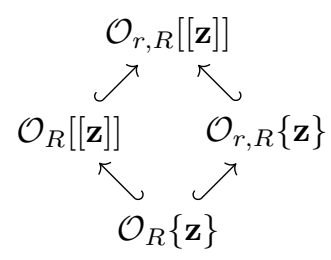

Figure 3: Diagram of inclusions

Proof. The Maximum Modulus Principle for analytic functions guarantees that the $\sup _{A}\|f\|$ is attained on the boundary of $A$. As $\partial D_{r, R} \subset \overline{D_{R}}$ and $\partial D_{R} \subset$ $\partial D_{r, R}$, it follows that $\sup _{D_{r, R}}\|f\|=\sup _{D_{R}}\|f\|$.

of Lemma 4. By the claim above, as $f \in \mathcal{O}_{r, R}[[\mathbf{z}]]$, each coefficient $f_{K}$ satisfies

$$
\sup _{D_{R^{\prime}}}\left\|f_{K}\right\|=\sup _{D_{r^{\prime}, R^{\prime}}}\left\|f_{K}\right\| \leq C M^{K}
$$

for any $0<r^{\prime}<R^{\prime}<R$. Consequently, $f \in \mathcal{O}_{R}\{\mathbf{z}\}$. The reciprocal is immediate.

\subsection{Automorphisms and Derivations.}

A $\mathbb{C}$-linear endomorphism of $\mathcal{O}_{r, R}[[\mathbf{z}]]$ is defined by a sequence indexed by $\mathbb{N}$ of $\mathbb{C}$-linear maps

$$
\Phi^{(i)} \in \operatorname{Hom}_{\mathbb{C}}\left(\mathcal{O}_{r, R}[[\mathbf{z}]], J^{(i)}\right)
$$

such that, for each $j<i, \pi_{j i} \circ \Phi^{(i)}=\Phi^{(j)}$, where $\pi_{j i}$ is a bonding map, and

$$
\Phi(f)=\prod_{i \in \mathbb{N}} \Phi^{(i)}(f) .
$$

We denote by $\operatorname{End}_{\mathbb{C}}\left(\mathcal{O}_{r, R}[[\mathbf{z}]]\right)$ the set of such endomorphisms, and we say that $\Phi^{(i)}$ is the $i^{t h}$-truncation of $\Phi$.

A change of coordinates in $\mathcal{O}_{r, R}[[\mathbf{z}]]$ can be seen as a linear automorphism that preserves $\mathfrak{m}$. That is, an invertible endomorphism $\Phi \in \operatorname{End}_{\mathbb{C}}\left(\mathcal{O}_{r, R}[[\mathbf{z}]]\right)$, satisfying

1. $\Phi(f . g)=\Phi(f) . \Phi(g), \forall f, g \in \mathcal{O}_{r, R}[[\mathbf{z}]]$.

2. $\Phi(\mathfrak{m}) \subset \mathfrak{m}$.

We denote by $\mathcal{A}\left(\mathcal{O}_{r, R}[[\mathbf{z}]]\right)$ the group of such automorphisms in $\mathcal{O}_{r, R}[[\mathbf{z}]]$, and we say that $\Phi \in \mathcal{A}\left(\mathcal{O}_{r, R}[[\mathbf{z}]]\right)$ is a $D_{r, R}$-transversely formal automorphism

Similarly, a vector field with components in $\mathcal{O}_{r, R}[[\mathbf{z}]]$ can be seen as a derivation on this ring. That is, an endomorphism $X \in \operatorname{End}_{\mathbb{C}}\left(\mathcal{O}_{r, R}[[\mathbf{z}]]\right)$, satisfying the Leibniz's rule

$$
X(f . g)=X(f) . g+X(g) . f \quad \forall f, g \in \mathcal{O}_{r, R}[[\mathbf{z}]] .
$$


We denote by $\mathcal{D}\left(\mathcal{O}_{r, R}[[\mathbf{z}]]\right)$ the $\mathcal{O}_{r, R}[[\mathbf{z}]]$-module of such derivations that has a structure of Lie Algebra with the Lie Bracket given by

$$
[X, Y]=X \circ Y-Y \circ X .
$$

An element $X \in \mathcal{D}\left(\mathcal{O}_{r, R}[[\mathbf{z}]]\right)$ is called $D_{r, R}$-transversely formal derivation.

Definition 3. A sequence $\left\{\Phi_{k}\right\}_{k \in \mathbb{N}}$ of endomorphism in $\mathcal{O}_{r, R}[[\mathbf{z}]]$ is called summable if for each $j \in \mathbb{N}$ there exists a natural number $K=K(j)$ such that the $j^{\text {th }}$-truncation of $\Phi_{k}$ is zero for all $k \geq K$.

Lemma 5. A summable sequence of endomorphism $\left\{\Phi_{k}\right\}_{k \in \mathbb{N}}$ in $\mathcal{O}_{r, R}[[\mathbf{z}]]$ defines an endomorphism $\Psi:=\sum_{k=0}^{\infty} \Phi_{k}$.

Proof. By the definition of summable sequence, each $\Psi^{(j)}=\sum_{k=0}^{\infty} \Phi_{k} \bmod \mathfrak{m}^{j+1}$ is a finite sum of terms, and for $j<i \in \mathbb{N}$

$$
\begin{aligned}
\pi_{j i} \Psi^{(i)} & =\pi_{j i} \sum_{k=0}^{\infty} \Phi_{k} \quad \bmod \mathfrak{m}^{i+1} \\
& =\sum_{k=0}^{\infty} \Phi_{k} \quad \bmod \mathfrak{m}^{j+1}=\Psi^{(j)}
\end{aligned}
$$

We are particular interested in the case where a sequence of endomorphism is defined by successive powers $X^{k}=\underbrace{X \circ \cdots \circ X}_{k}$ of a given derivation $X$, where $X^{k}(f)$ means applying the derivative $X k$-times on $f \in \mathcal{O}_{r, R}[[\mathbf{z}]]$.

Definition 4. A vector field $X \in \mathcal{D}\left(\mathcal{O}_{r, R}[[\mathbf{z}]]\right)$ is called nilpotent if $X$ preserves the ideal $\mathfrak{m}$, i.e. $X(\mathfrak{m}) \subset \mathfrak{m}$, and for all $j \in \mathbb{N}$ exists $N=N(j) \in \mathbb{N}$ such that

$$
X^{N}\left(\mathfrak{m}^{j}\right) \subset \mathfrak{m}^{j+1}
$$

where $\mathfrak{m}^{0}=\mathcal{O}_{r, R}[[z]]$. We denote by $\mathcal{N}\left(\mathcal{O}_{r, R}[[\mathbf{z}]]\right) \subset \mathcal{D}\left(\mathcal{O}_{r, R}[[\mathbf{z}]]\right)$ the set of $D_{r, R}$-transversely formal nilpotent vector fields.

Proposition 6. Let $X \in \mathcal{N}\left(\mathcal{O}_{r, R}[[\mathbf{z}]]\right)$. For any sequence $\left\{c_{k}\right\}_{k \in \mathbb{N}}$ of complex numbers, the sequence $\left\{c_{k} X^{k}\right\}_{k \in \mathbb{N}}$ is summable.

Proof. By the definition, there exist $N(0), N(1), \ldots, N(j) \in \mathbb{N}$, such that

$$
X^{N(0)}\left(\mathfrak{m}^{0}\right) \subset \mathfrak{m}, \quad X^{N(1)}(\mathfrak{m}) \subset \mathfrak{m}^{2}, \ldots X^{N(j)}\left(\mathfrak{m}^{j}\right) \subset \mathfrak{m}^{j+1} .
$$

Then,

$$
X^{N(0)+\cdots+N(j)}\left(\mathfrak{m}^{0}\right) \subset \mathfrak{m}^{j+1}
$$

which implies that the sequence $\left\{c_{k} X^{k}\right\}_{k \in \mathbb{N}}$ is summable. 
Corollary 7. Let $X \in \mathcal{N}\left(\mathcal{O}_{r, R}[[\mathbf{z}]]\right)$. Then, for all $t \in \mathbb{C}$, the sequence $\left\{\frac{t^{k}}{k !} X^{k}\right\}_{k \in \mathbb{N}}$ defines an endomorphism $\exp (t X)=\sum_{k=0}^{\infty} \frac{t^{k}}{k !} X^{k}$ called the time $t$ exponential.

Proof. Making $c_{k}=\frac{t^{k}}{k !}$, we conclude from Proposition 6 that the series $\sum_{k=0}^{\infty} \frac{t^{k}}{k !} X^{k}$ converges.

A corollary of the proof of Proposition 6 is the following.

Corollary 8. For all $i \in \mathbb{N}$, the $i^{\text {th }}$-truncation of the automorphism $\exp (t X)$ is polynomial in $t \in \mathbb{C}$ with coefficients in $\mathcal{O}_{r, R}$. In other words, for $f \in \mathcal{O}_{r, R}[[\mathbf{z}]]$, $\exp (t X)(f)=\left(\sum_{k=0}^{\infty} \frac{t^{k}}{k !} X^{k}(f)\right) \quad \bmod \mathfrak{m}^{i+1}$ belongs to $\mathcal{O}_{r, R}[t][[\mathbf{z}]]$.

In order to study the invertibility of the exponential map, we consider automorphisms and derivations satisfying some flatness conditions. We say that a $D_{r, R}$-transversely formal automorphism $\Phi \in \mathcal{A}\left(\mathcal{O}_{r, R}[[\mathbf{z}]]\right)$ is tangent to the identity to order $k$ if $\Phi(x)=x \bmod \mathfrak{m}^{k+1}$ and $\Phi\left(z_{i}\right)=z_{i} \bmod \mathfrak{m}^{k+1}$ for all $i \in\{1, \ldots, n\}$. We denote by $\mathcal{A}_{k}\left(\mathcal{O}_{r, R}[[\mathbf{z}]]\right)$ the subset of such automorphisms.

A vector field $X=a(x, \mathbf{z}) \partial_{x}+\sum_{j=1}^{n} b_{j}(x, \mathbf{z}) \partial_{z_{j}} \in \mathcal{N}\left(\mathcal{O}_{r, R}[[\mathbf{z}]]\right)$ is $k$-flat if $a(x, \mathbf{z}) \in \mathfrak{m}^{k}, b_{i}(x, \mathbf{z}) \in \mathfrak{m}^{k+1}$ for all $i \in\{1, \ldots, n\}$. We denote by $\mathcal{N}_{k}\left(\mathcal{O}_{r, R}[[\mathbf{z}]]\right)$ for the subset of such derivations.

Proposition 9. ([Ser09], Theorem 7.2) For each integer number $k \geq 1$, the exponential map $\exp : \mathcal{N}_{k}\left(\mathcal{O}_{r, R}[[\mathbf{z}]]\right) \rightarrow \mathcal{A}_{k}\left(\mathcal{O}_{r, R}[[\mathbf{z}]]\right) ; X \mapsto \exp (X)$ is one-toone with inverse given by, $\log (\Phi)=\sum_{n=0}^{\infty} \frac{(-1)^{n+1}}{n}(\Phi-i d)^{n}$.

\subsection{The Lie Brackets and the Exponential map.}

In this subsection, we list some results related to the exponential map which are going to be used later. After that, we show the connection between symmetries for a vector field and its centralizer.

In the next propositions, we consider $\Phi \in \mathcal{A}\left(\mathcal{O}_{r, R}[[\mathbf{z}]]\right), X, Y \in \mathcal{N}\left(\mathcal{O}_{r, R}[[\mathbf{z}]]\right)$, and $Z, W \in \mathcal{D}\left(\mathcal{O}_{r, R}[[\mathbf{z}]]\right)$.

Proposition 10. The following properties hold

1. $\Phi^{*}[Z, W]=\left[\Phi^{*} Z, \Phi^{*} W\right]$, where $\Phi^{*} Z=\Phi \circ Z \circ \Phi^{-1}$.

2. $[Z, f W]=f[Z, W]+Z(f) W$, for $f \in \mathcal{O}_{r, R}[[\mathbf{z}]]$.

3. If $[X, Y]=0$, then $\exp (X+Y)=\exp X$. $\exp Y$.

Proof. The properties (1) and (2) follow directly from the Lie Bracket's definition, and (3) follows from the Newton's Binomial.

Remark 1. Since $\exp 0=i d$ and $[X,-X]=0$, the item (3) implies that $(\exp X)^{-1}=\exp -X$ and $\exp (n X)=\exp ^{n} X$, for all $n \in \mathbb{Z}$. 
The two maps $A d_{X}, a d_{Z}: \mathcal{D}\left(\mathcal{O}_{r, R}[[\mathbf{z}]]\right) \rightarrow \mathcal{D}\left(\mathcal{O}_{r, R}[[\mathbf{z}]]\right)$ defined by

$$
\begin{gathered}
\operatorname{ad}_{Z}(W)=[Z, W], \\
A d_{X}(W)=\exp X \circ W \circ(\exp X)^{-1},
\end{gathered}
$$

are connected by the following well-known result.

Proposition 11. For a fixed $t \in \mathbb{C}, \exp \left(a d_{t X}\right) Z=A d_{t X} Z$.

Proof. We give a short proof for the sake of completeness. It is enough to show that

$$
a d_{X}^{n} Z=\sum_{k=0}^{n}\left(\begin{array}{l}
n \\
k
\end{array}\right) X^{k} \circ Z \circ(-X)^{n-k} .
$$

We use induction to prove it. The base case $n=1$ is clear, let us assume that it is true for $n=m$. By hypothesis of induction

$$
\begin{aligned}
a d_{X}^{m+1} Z & =a d_{X}\left(\sum_{k=0}^{m}\left(\begin{array}{c}
m \\
k
\end{array}\right) X^{k} \circ Z \circ(-X)^{m-k}\right) \\
& =\sum_{k=0}^{m}\left(\begin{array}{c}
m \\
k
\end{array}\right) X^{k+1} \circ Z \circ(-X)^{m-k}-\sum_{k=0}^{m}\left(\begin{array}{c}
m \\
k
\end{array}\right) X^{k} \circ Z \circ(-X)^{m-k} X \\
& =\sum_{k=0}^{m}\left(\begin{array}{c}
m \\
k
\end{array}\right) X^{k+1} \circ Z \circ(-X)^{m-k}+\sum_{k=0}^{m}\left(\begin{array}{c}
m \\
k
\end{array}\right) X^{k} \circ Z \circ(-X)^{m-k+1},
\end{aligned}
$$

By the Pascal's triangle

$$
\begin{aligned}
\sum_{k=0}^{m}\left(\begin{array}{c}
m \\
k
\end{array}\right) X^{k+1} & \circ Z \circ(-X)^{m-k}+\sum_{k=0}^{m}\left(\begin{array}{c}
m \\
k
\end{array}\right) X^{k} \circ Z \circ(-X)^{m-k+1}= \\
& =\sum_{k=0}^{m+1}\left(\begin{array}{c}
m+1 \\
k
\end{array}\right) X^{k} \circ Z \circ(-X)^{m-k} .
\end{aligned}
$$

Definition 5. A $D_{r, R}$-transversely formal symmetry for $Z \in \mathcal{D}\left(\mathcal{O}_{r, R}[[\mathbf{z}]]\right)$ is an automorphism $\Phi \in \mathcal{A}\left(\mathcal{O}_{r, R}[[\mathbf{z}]]\right)$ such that

$$
\Phi \circ Z \circ \Phi^{-1}=Z \text {. }
$$

We remark that we can similarly define a formal symmetry for a derivation in $\mathcal{D}(\mathbb{C}[[x, \mathbf{z}]])$. In addition, Proposition 9 has its version for automorphisms and derivations over $\mathbb{C}[[x, \mathbf{z}]]$. Then, all the following results of this subsection have their respective statements over the formal objects. 
Definition 6. A $D_{r, R}$-transversely formal automorphism $\Phi$ is said to be $x$ normalized if it is $\mathcal{O}_{r, R}$-linear and has the form

$$
\Phi(x, \mathbf{z})=\left(x, \sum_{i=1}^{n} a_{1 i} z_{i}+\phi_{1}(x, \mathbf{z}), \ldots, \sum_{i=1}^{n} a_{n i} z_{i}+\phi_{n}(x, \mathbf{z})\right),
$$

where $\left(a_{i j}\right)_{n \times n}$ is an invertible constant matrix and $\phi_{1}, \ldots, \phi_{n} \in \mathfrak{m}^{2} \subset \mathcal{O}_{r, R}[[\mathbf{z}]]$. We denote by $\mathcal{A}_{\text {norm }}\left(\mathcal{O}_{r, R}[[\mathbf{z}]]\right)$ the set of such automorphisms.

Supposing that the maps $\phi_{1}, \ldots, \phi_{n}$ in Definition 6 lie in the respective rings $\mathcal{O}_{R}[[\mathbf{z}]]$ and $\mathbb{C}[[x, \mathbf{z}]]$, we define similarly $\mathcal{A}_{\text {norm }}\left(\mathcal{O}_{R}[[\mathbf{z}]]\right)$ and $\mathcal{A}_{\text {norm }}(\mathbb{C}[[x, \mathbf{z}]])$.

In the next result, we use the fact that any $x$-normalized automorphism $\Phi \in \mathcal{A}_{\text {norm }}\left(\mathcal{O}_{r, R}[[\mathbf{z}]]\right)$ can be uniquely decomposed as $\Phi=A \circ \Psi$, where $A$ is an invertible linear change of coordinates in the indeterminates $x, z_{1}, \ldots, z_{n}$, i.e.,

$$
\begin{aligned}
A x & =x, \\
A z_{i} & =\sum_{j=1}^{n} a_{i j} z_{j}, \quad a_{i j} \in \mathbb{C}
\end{aligned}
$$

and $\Psi$ is a $D_{r, R}$-transversely formal automorphism tangent to the identity to order 1. Using Proposition 9, we can write

$$
\Phi=A \circ \exp Z,
$$

for a uniquely determined $D_{r, R}$-transversely formal nilpotent vector field $Z$. We say that this decomposition is the exponential decomposition of $\Phi$.

Lemma 12. Let $X \in \mathcal{D}(\mathbb{C}[[x, \mathbf{z}]])$ be a linear derivation and $\Phi$ an $x$-normalized $D_{r, R}$-transversely automorphism. If $\Phi$ is a $D_{r, R}$-transversely formal symmetry for $X$, and $\Phi=A \circ \exp Z$ is the exponential decomposition of $\Phi$, then the automorphisms $A$ and $\exp Z$ are $D_{r, R}$-transversely formal symmetries for $X$.

Proof. Note that the linear part of $\Phi^{*} X$ is $A^{*} X$. As $X$ is linear and $\Phi$ is a symmetry for $X$, we have $A^{*} X=X$. On the other hand, $X=\Phi^{*} X=$ $\exp Z^{*}\left(A^{*} X\right)=\exp Z^{*} X$, then $\exp Z$ is also a symmetry for $X$.

For the following sequence of results, consider $X \in \mathcal{N}\left(\mathcal{O}_{r, R}[[\mathbf{z}]]\right)$ and $Z \in$ $\mathcal{D}\left(\mathcal{O}_{r, R}[[\mathbf{z}]]\right)$.

Lemma 13. Let $\exp X$ be a $D_{r, R}$-transversely formal symmetry for $Z$. Then, $\exp t X$ is a $D_{r, R}$-transversely formal symmetry for $Z$ for all $t \in \mathbb{C}$.

Proof. The following argument is based on the proof of Lemma 2.4.9 Dia19. By Corollary 8 , we know that for all $i \in \mathbb{N}$ the $i^{t h}$-truncation of $\exp t X$ is polynomial on $t$, so the map

$$
P_{k}(t, x, \mathbf{z})=(\exp t X \circ Z \circ \exp -t X-Z) \bmod \mathfrak{m}^{k+1}
$$

is as well. 
By the Remark $1 . \exp n X=(\exp X)^{n}$ for all $n \in \mathbb{N}$. All these facts together imply that

$$
\begin{aligned}
\exp n X \circ Z \circ \exp -n X= & \exp ^{n} X \circ Z \circ(\exp X)^{-n} \\
& =\exp ^{n-1} X \circ\left(\exp X \circ Z \circ(\exp X)^{-1}\right) \circ(\exp X)^{-n+1} \\
& =\exp ^{n-1} X \circ Z \circ(\exp X)^{-n+1} \\
& \vdots \\
& =Z
\end{aligned}
$$

In other words, for all $n, k \in \mathbb{N}$, the polynomial $P_{k}(n, x, \mathbf{z})$ vanishes. As consequence, $P(t, x, \mathbf{z})=0$, therefore, $\exp t X \circ Z \circ \exp -t X=Z$ for all $t \in \mathbb{C}$. The reciprocal is obvious.

Lemma 14. For all $t \in \mathbb{C}$, the exponential map $\exp t X$ is a $D_{r, R}$-transversely formal symmetry for $Z$ if only if $[X, W]=0$.

Proof. Assume that the $\exp t X$ is a $D_{r, R}$-transversely formal symmetry. The Proposition 11 implies

$$
Z=A d_{t X} Z=\exp \left(a d_{t X}\right) Z=\left(Z+t[X, Z]+\frac{t^{2}}{2}[X,[X, Z]]+\ldots\right) .
$$

Taking the derivative on $t$ and evaluating on zero, we find $[X, Z]=0$. The reciprocal is obvious.

Proposition 15. The map $\exp X$ is a $D_{r, R}$-transversely formal symmetry for $Z$ if only if $X$ commutes with $Z$, i.e. $[X, Z]=0$.

Proof. By Lemma $13, \exp X$ is a $D_{r, R}$-transversely formal symmetry for $Z$ if only if for all $t \in \mathbb{C}, \exp t X$ is also a $D_{r, R}$-transversely formal symmetry for $Z$. By Lemma 14 the $\exp t X$ is a $D_{r, R}$-transversely formal symmetry for $Z$ for all $t \in \mathbb{C}$ if only if $[X, Z]=0$.

\subsection{Normal Form of $x$-normalized $D_{R}$-transversely Formal Vector Fields.}

In this subsection, we adapt the Normal Formal Theory of Poincaré (see for instance [Mar81]) to our present setting of $D_{R}$-transversely formal vector fields.

We denote by $\mathcal{D}_{\text {norm }}\left(\mathcal{O}_{R}[[\mathbf{z}]]\right)$ the set of $x$-normalized derivations in $\mathcal{D}\left(\mathcal{O}_{R}[[\mathbf{z}]]\right)$. We recall that an element $X \in \mathcal{D}_{\text {norm }}\left(\mathcal{O}_{R}[[\mathbf{z}]]\right)$ can be written in the following form

$$
x \partial_{x}+\sum_{i=1}^{n} \sum_{j=1}^{n} a_{i j} z_{j} \partial_{z_{i}}+\sum_{i=1}^{n} b_{i}(x, \mathbf{z}) \partial_{z_{i}}
$$

where $a_{i j} \in \mathbb{C}$ and $b_{i}(x, \mathbf{z}) \in \mathfrak{m}^{2} \subset \mathcal{O}_{R}[[\mathbf{z}]]$.

In the next proof, we use the graded lexicographic order $>_{\text {gr.lex }}$ on $\mathbb{Z}^{n}$ defined as follows. For $K=\left(k_{1}, \ldots, k_{n}\right)$ and $L=\left(l_{1}, \ldots, l_{n}\right)$ two $n$-uples in $\mathbb{Z}^{n}$, we write 
$K>_{\text {gr.lex }} L$ if either $k_{1}+\cdots+k_{n}=:|K|>|L|:=l_{1}+\cdots+l_{n}$, or $|K|=|L|$ and $\left(k_{1}, \ldots, k_{n}\right)>_{\text {lex }}\left(l_{1}, \ldots, l_{n}\right)$. We say that $K$ is greater than $L$ when $K>_{\text {gr.lex }} L$.

In order to simplify the notation, we denote by $L(\lambda)$ the diagonal vector field $\sum_{i=1}^{n} \lambda_{i} z_{i} \partial_{z_{i}}$, where $\lambda=\left(\lambda_{1}, \ldots, \lambda_{n}\right) \in \mathbb{C}^{n}$.

Proposition 16. For any $X \in \mathcal{D}_{\text {norm }}\left(\mathcal{O}_{R}[[\mathbf{z}]]\right)$ with $1, \mu_{1}, \ldots, \mu_{n}$ as eigenvalues there exists an $x$-normalized $D_{R}$-transversely formal change of coordinates which conjugates $X$ to a vector field of the form

$$
x \partial_{x}+L(\mu)+\sum_{i=2}^{n} \epsilon_{i} z_{i}^{-1} z_{i-1} L\left(e_{i}\right)+\sum_{K} x^{-\langle\mu, K\rangle} \mathbf{z}^{K} L\left(\lambda_{K}\right),
$$

where $\mu=\left(\mu_{1}, \ldots, \mu_{n}\right), \epsilon_{i} \in\{0,1\}$ is nonzero only if $\mu_{i}=\mu_{i-1}$, the last sum on the right-hand side is taken over all indices $K \in \mathcal{L}_{n, 1}$ such that $\langle\mu, K\rangle \in \mathbb{Z}_{\leq 0}$.

Proof. Applying the usual Jordan Normal Theory, we can assume that the linear part of $X$ has the form

$$
X_{\text {lin }}=x \partial_{x}+L(\mu)+\sum_{i=2}^{n} \epsilon_{i} z_{i}^{-1} z_{i-1} L\left(e_{i}\right),
$$

where $\mu=\left(\mu_{1}, \ldots, \mu_{n}\right), \epsilon_{i} \in\{0,1\}$ is nonzero only if $\mu_{i}=\mu_{i-1}$.

We can write the series expansion of $X$ as

$$
X_{l i n}+\sum_{K, j} g_{K, j}(x) \mathbf{z}^{K} L\left(e_{j}\right),
$$

where the last sum on the right-hand side is taken over all indices $j \in\{1, \ldots, n\}$ and $K \in \mathcal{L}_{n, 1}$, and $g_{K, j} \in \mathcal{O}_{R}$. We say that a nonzero term $g_{K, j}(x) \mathbf{z}^{K} L\left(e_{j}\right)$ in (4) is resonant if $\langle\mu, K\rangle$ lies in $\mathbb{Z}_{\leq 0}$ and $g_{K, j}(x)=\lambda_{K, j} x^{-\langle\mu, K\rangle}$, where $\lambda_{K, j} \in \mathbb{C}$.

We eliminate the nonresonant terms by successive applications of automorphisms of the form

$$
\Phi=\exp \left(f(x) \mathbf{z}^{K} L\left(e_{j}\right)\right),
$$

where $f \in \mathcal{O}_{R}, K \in \mathcal{L}_{n, 1}$, and $j \in\{1, \ldots, n\}$ are conveniently chosen.

Indeed, we consider the smallest nonresonant term $g_{K_{0}, j_{0}}(x) \mathbf{z}^{K_{0}} L\left(e_{j_{0}}\right)$ in (4) with respect to the pair $(K, j)$ and to the gr.lex order. The action of $\Phi=$ $\exp \left(f(x) \mathbf{z}^{K_{0}} L\left(e_{j_{0}}\right)\right)$ by conjugation on $X$ gives the expression

$$
\begin{aligned}
\Phi^{*} X= & X_{l i n}+\sum_{(K, j) \leq\left(K_{0}, j_{0}\right)} \lambda_{K, j} x^{-\langle\mu, K\rangle} \mathbf{z}^{K} L\left(e_{j}\right)+ \\
& +\left(g_{K_{0}, j_{0}}(x)-\left(x \partial_{x}-\left\langle\mu, K_{0}\right\rangle\right) f(x)\right) \mathbf{z}^{K_{0}} L\left(e_{j_{0}}\right)+\mathcal{R},
\end{aligned}
$$

where the rest term $\mathcal{R}$ is a sum of vector fields $g_{K, j} \mathbf{z}^{K} L\left(e_{j}\right)$ with $(K, j)>_{\text {gr.lex }}$ $\left(K_{0}, j_{0}\right)$.

Writing the series expansion $g_{K_{0}, j_{0}}(x)=\sum_{i \neq\left\langle\mu, K_{0}\right\rangle} a_{i} x^{i}+a_{\left\langle\mu, K_{0}\right\rangle} x^{\left\langle\mu, K_{0}\right\rangle}$, with the convention that $a_{\left\langle\mu, K_{0}\right\rangle}=0$ if $\left\langle\mu, K_{0}\right\rangle \in \mathbb{Z}_{\leq 0}$, we can define $f(x)=$ $\sum_{i \neq\left\langle\mu, K_{0}\right\rangle} \frac{a_{i}}{i+\left\langle\mu, K_{0}\right\rangle} x^{i}$. As a result, we obtain a new vector field whose smallest nonresonant term is of order strictly greater than $\left(K_{0}, j_{0}\right)$. 
We say that the expression (3) is a normal form for $X$.

Proposition 17. Let $X, Y \in \mathcal{D}_{\text {norm }}\left(\mathcal{O}_{R}[[\mathbf{z}]]\right)$ and $\Psi \in \mathcal{A}_{\text {norm }}(\mathbb{C}[[x, \mathbf{z}]])$. If $\Psi$ conjugates $X$ to $Y$, then $\Psi \in \mathcal{A}_{\text {norm }}\left(\mathcal{O}_{R}[[\mathbf{z}]]\right)$.

Proof. By Proposition 16 , there exist $\Phi_{1}, \Phi_{2} \in \mathcal{A}_{\text {norm }}\left(\mathcal{O}_{R}[[\mathbf{z}]]\right)$ which diagonalize the respective semisimple parts of $X$ and $Y$, in other words, we have the respective Jordan decompositions

$$
\begin{gathered}
\Phi_{1}^{*} X=X_{s}+X_{n}=x \partial_{x}+L(\mu)+X_{n}, \\
\Phi_{2}^{*} Y=Y_{s}+Y_{n}=x \partial_{x}+L(\lambda)+Y_{n} .
\end{gathered}
$$

By hypothesis, there exists $\Psi \in \mathcal{A}_{\text {norm }}(\mathbb{C}[[x, \mathbf{z}]])$ such that

$$
\Psi^{*} X=Y
$$

which implies $\mu=\lambda$. By the uniqueness of the Jordan decomposition, the automorphism $\Psi_{0}=\Phi_{2} \circ \Psi \circ \Phi_{1}^{-1} \in \mathcal{A}_{\text {norm }}(\mathbb{C}[[x, \mathbf{z}]])$ is such that

$$
\Psi_{0}^{*}\left(x \partial_{x}+L(\mu)\right)=x \partial_{x}+L(\mu) .
$$

It means that $\Psi_{0}$ is an $x$-normalized formal symmetry for $x \partial_{x}+L(\mu)$.

Consider the exponential decomposition $A \circ \exp Z$ of $\Psi_{0}$. Since $\Psi_{0}$ is $x$ normalized, $Z$ has the form $\sum_{K \in \mathcal{L}_{n, 1}} b_{K}(x) \mathbf{z}^{K} L\left(\lambda_{K}\right)$, where each $b_{K} \in \mathbb{C}[[x]]$. By Lemma 12, we know that $\exp Z$ is a symmetry for $x \partial_{x}+L(\mu)$. And by Proposition 15, we have that

$$
\left[Z, x \partial_{x}+L(\mu)\right]=0 .
$$

Using the above expansion of $Z$, this equality is equivalent to state that

$$
\left(x \partial_{x}+\langle\mu, K\rangle\right) b_{K}(x)=0
$$

for all $K \in \mathcal{L}_{n, 1}$. Writing $b_{K}(x)=\sum_{i=0}^{\infty} c_{i} x^{i}$, with $c_{i} \in \mathbb{C}$, we obtain for each $i \in \mathbb{N}$

$$
(i+\langle\mu, K\rangle) c_{i}=0 .
$$

This implies that either $\langle\mu, K\rangle \notin \mathbb{Z}_{<0}$ and $b_{K}=0$ or else $b_{K}(x)=c x^{-\langle\mu, K\rangle}$ for some constant $c \in \mathbb{C}$. In other words, $Z$ is a $D_{R}$-transversely formal vector field, and consequently, $\exp Z$ is a $D_{R}$-transversely formal change of coordinates. In conclusion, $\Phi=\Phi_{2}^{-1} \circ \Psi_{0} \circ \Phi_{1}$ is a $D_{R}$-transversely formal automorphism.

The property of no transverse negative resonance stated in the introduction can be reformulated as follows.

Definition 7. We say that a vector field $X \in \mathcal{D}\left(\mathcal{O}_{R}[[\mathbf{z}]]\right)$ with $1, \mu_{1}, \ldots, \mu_{n}$ as eigenvalues has no transverse negative resonance if

$$
\left\langle\mu, \mathcal{L}_{n, 0}\right\rangle \cap \mathbb{Z}_{\geq 1}=\emptyset,
$$

where $\mu=\left(\mu_{1}, \ldots, \mu_{n}\right)$. 
Now, we have all the tools to prove the Theorem 3 . Recall that it characterizes the center of an $x$-normalized $D_{R}$-transversely formal vector field with no transverse negative resonance on the set of $x$-normalized $D_{r, R^{-}}$-transversely formal vector fields.

of Theorem 3. By Proposition 16, we can assume that $X$ has the form

$$
X=x \partial_{x}+L(\mu)+\sum_{i=2}^{n} \epsilon_{i} z_{i}^{-1} z_{i+1} L\left(e_{i}\right)+\sum_{K} b_{K} x^{-\langle\mu, K\rangle} \mathbf{z}^{K} L\left(\lambda_{K}\right) .
$$

Since $Y$ is $x$-normalized, it can be expanded as

$$
Y=x \partial_{x}+\sum_{i=1}^{n} \sum_{j=1}^{n} c_{i j} z_{j} \partial_{z_{i}}+\sum_{l, K} d_{l K} x^{l} \mathbf{z}^{K} L\left(\lambda_{K}\right),
$$

where $l \in \mathbb{Z}, K \in \mathcal{L}_{n, 1}$.

As $Y$ commutes with $X$, it has to commute with its semisimple part $x \partial_{x}+$ $L(\mu)$. This implies that for each $l \in \mathbb{Z}, K \in \mathcal{L}_{n, 1}$, we must have

$$
(l+\langle\mu, K\rangle) d_{l K} x^{l} \mathbf{z}^{K} L\left(\lambda_{K}\right)=\left[x \partial_{x}+L(\mu), d_{l K} x^{l} \mathbf{z}^{K} L\left(\lambda_{K}\right)\right]=0 .
$$

Since $X$ has no transverse negative resonance, both expressions can vanish only if $l \geq 0$. This means all the monomials on the expansion of $Y$ have positive exponents on $x$. Consequently $Y \in \mathcal{D}_{\text {norm }}\left(\mathcal{O}_{R}[[\mathbf{z}]]\right)$.

\section{The Crossing Type Foliations}

The first goal of this section is to define the holonomy for a crossing type foliation. Then, we show that it is always possible to construct an $x$-normalized automorphism that conjugates local generators of crossing type foliations on a neighborhood of $D_{r, R}$ if the conditions $|(a)|,(b)$ and $\mid(c)$ (see section 1 ) are satisfied. Using these results, we prove that is always possible to extend this automorphism to a neighborhood of the origin. In other words, we prove the main result of this paper. After that, we show an application in dimension 3.

We will need the following well-known result.

Lemma 18. Let $X \in \mathcal{D}\left(\mathcal{O}_{R}\{\boldsymbol{z}\}\right)$ be a vector field having the form

$$
X=x \partial_{x}+\sum_{i=1}^{n} \sum_{j=1}^{n} a_{i j} z_{j} \partial_{z_{i}}+\sum_{i=1}^{n} \sum_{j=1}^{n} b_{i j}(x) z_{i} \partial_{z_{j}},
$$

where $a_{i j} \in \mathbb{C}$, and $b_{i j} \in \mathcal{O}_{R}$ vanishes at $0 \in \mathbb{C}$. If $X$ has no transverse negative resonance, then, possibly reducing the radius $R$, there exists an $x$-normalized change of coordinates which conjugates $X$ to a vector field with the form

$$
x \partial_{x}+\sum_{i=1}^{n} \sum_{j=1}^{n} a_{i j} z_{j} \partial_{z_{i}} .
$$


Proof. Let $1, \mu_{1}, \ldots, \mu_{n}$ be the eigenvalues of $X$. The hypothesis of no transverse negative resonance implies that $\mu_{i}-\mu_{j} \notin \mathbb{Z}_{\geq 1}$, for any $i \neq j$. Hence, the expansion (5) has no resonant term in the sense of Poincaré. Now, note that the vector field $X$ is associated to the following differential system

$$
\left\{\begin{array}{l}
\dot{x}=x, \\
\dot{\mathbf{z}}=\left(A_{0}+x A_{1}+\ldots\right) \mathbf{z},
\end{array}\right.
$$

where $A_{0}=\left(a_{i j}\right)_{n \times n}$, and $A_{i}$ is a n-dimensional square complex matrix for all $i \in \mathbb{Z}_{>0}$. Then, by Theorems 16.15 and 16.16 in [Y08, there exists an xnormalized change of coordinates which conjugates $X$ to the vector field $x \partial_{x}+$ $A_{0} \mathbf{z}$.

Lemma 19. Let $(\mathcal{F}, H, \Gamma)$ be a crossing type foliation which its local generators have no transverse negative resonance. Then, there exist local coordinates

$$
(x, \mathbf{z})=\left(x, z_{1}, \ldots, z_{n}\right)
$$

such that $\Gamma:=\{\mathbf{z}=0\}, H:=\{x=0\}$. Moreover, once these coordinates are fixed, there exists a unique local generator $X$ for $\mathcal{F}$ that is an $x$-normalized $D_{R}$-transversely convergent vector field.

Proof. We can choose coordinates $(x, \mathbf{z})$ such that $\Gamma:=\{\mathbf{z}=0\}, H:=\{x=0\}$. The condition (ii.b) in the definition of crossing type foliation (see section 1 ) implies that a local generator for $\mathcal{F}$ can be written in these coordinates as

$$
Y_{0}=g(x, \mathbf{z}) x \partial_{x}+\sum_{i=1}^{n} h_{i}(x, \mathbf{z}) \partial_{z_{i}}
$$

where $g, h_{1}, \ldots$, and $h_{n}$ do not have common factors and $h_{1}, \ldots, h_{n}$ lie in the ideal $\mathfrak{m}$. The condition (iii.) says that the linear part of $Y_{0}$ has a nonzero eigenvalue in the $\Gamma$-direction. Then $g(0) \neq 0$, and $Y_{1}=g^{-1} Y$ is a local generator of $\mathcal{F}$.

By hypothesis, $Y_{1}$ has no transverse negative resonance, then, by Lemma 18 . there exists an $x$-normalized $D_{R}$-transversely convergent change of coordinates that conjugates $Y_{1}$ to

$$
X=x \partial_{x}+\sum_{i=1}^{n} \sum_{j=1}^{n} a_{i j} z_{j} \partial_{z_{i}}+\sum_{i=1}^{n} b_{i}(x, \mathbf{z}) \partial_{z_{i}},
$$

where $\left(a_{i j}\right)_{n \times n}$ is a constant matrix, and $b_{i} \in \mathfrak{m}^{2} \subset \mathcal{O}_{R}\{\mathbf{z}\}$ for $1 \leq i \leq n$.

We say that the coordinates given by this lemma are adapted to the crossing type foliation $(\mathcal{F}, H, \Gamma)$ and that $X$ is the $x$-normalized local generator for $\mathcal{F}$ in these adapted coordinates.

For any $R>0$, up to a constant rescaling in the $x$ variable, we can suppose that the $x$-normalized local generators are defined in a domain containing a 
neighborhood of $\overline{D_{R} \times\{\mathbf{0}\}} \subset \mathbb{C}^{n+1}$. Then, we fix once for all an constant $R>1$.

In these adapted coordinates, the local $\Gamma$-holonomy can be computed by lifting the circular path $\left\{\left(e^{2 \pi \theta}, 0\right) ; \theta \in[0,1]\right\}$ along the leaves of the foliation that goes through a small poli-disk $\mathbb{D} \subset \mathbb{C}^{n}$ transverse to $\Gamma^{*}$ at the point $(1, \mathbf{0})$.

Definition 8. We say that $(\mathcal{F}, H, \Gamma)$ and $(\mathcal{G}, L, \Omega)$ are $D_{r, R}$-transversely equivalent if there exist $0<r<1<R$, respective adapted coordinates $(x, \mathbf{z})$ and $(y, \mathbf{w})$, and a bianalytic map $\Psi$ between two open neighborhoods $U, V \subset \mathbb{C}^{n+1}$ of $D_{r, R} \times\{\mathbf{0}\}$ such that $\Psi$ conjugates the $x$-normalized and $y$-normalized local generators restricted to $U$ and $V$, and we can write $\Psi$ in the form

$$
(y, \mathbf{w})=\left(x, \sum_{i=1}^{n} a_{1 i} z_{i}+\psi_{1}(x, \mathbf{z}), \ldots, \sum_{i=1}^{n} a_{n i} z_{i}+\psi_{n}(x, \mathbf{z})\right),
$$

where $\left(a_{i j}\right)_{n \times n}$ is an invertible constant matrix and $\psi_{1}, \ldots, \psi_{n} \in \mathfrak{m}^{2} \subset \mathcal{O}_{r, R}[[\mathbf{z}]]$.

We observe that $\Psi$ is an $x$-normalized $D_{r, R}$-transversely automorphism (see Definition 6).

Even though the next result is a simple application of Theorem 2 chapter IV CN85, we are going to give a prove of the new fact that the map which conjugates the local generators is $x$-normalized. We refer to CN77] for further details.

Proposition 20. Let $(\mathcal{F}, H, \Gamma)$ and $(\mathcal{G}, L, \Omega)$ be two crossing type foliations with respective local generators $X$ and $Y$ verifying (a), (b), and (c) (see section 11 ). Then, the crossing type foliations are $D_{r, R}$-transversely equivalent.

Proof. Let $K \subset \Gamma^{*}$ be a compact set containing the unity circle $\mathbb{S}^{1}$. Given a point $(x, \mathbf{z})$ in a convenient neighborhood of $K$, let $\alpha_{x}:[0,-\ln |x|] \rightarrow K$ and $\beta_{x}:[0, \tilde{t}] \rightarrow K$ be the curves such that $\alpha_{x}(t)=\left(x e^{t}, \mathbf{0}\right), \beta_{x}(t)=\left(x /|x| e^{-2 \pi i t}, \mathbf{0}\right)$, and $\beta_{x}(\tilde{t})=(1, \mathbf{0})$.

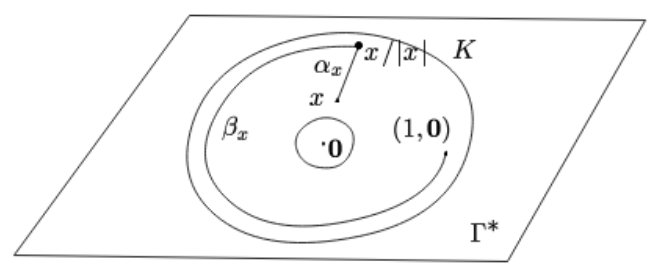

Figure 4: Composed path

Denote by $f_{\mathcal{F}}^{\gamma}$ and $f_{\mathcal{G}}^{\gamma}$ the respective lifts of a curve $\gamma \subset K$ to the leaves of $(\mathcal{F}, H, \Gamma)$ and $(\mathcal{G}, L, \Omega)$, and by $\varphi$ the map that conjugates the $\Gamma$-holonomy to the $\Omega$-holonomy. From Theorem 1.2 [CN77, we recall that the map that conjugates the local generators $X$ and $Y$ is given by

$$
\Psi(x, \mathbf{z}):=f_{\mathcal{G}}^{\alpha_{x}^{-1}} \circ f_{\mathcal{G}}^{\beta_{x}^{-1}} \circ \varphi \circ f_{\mathcal{F}}^{\beta_{x}} \circ f_{\mathcal{F}}^{\alpha_{x}}(x, \mathbf{z}) .
$$


By the hypothesis $(a)$, we can assume that $X \bmod \mathfrak{m}^{2}=Y \bmod \mathfrak{m}^{2}=x \partial_{x}+$ $\sum_{i=1}^{n} \sum_{j=1}^{n} a_{i j} z_{j} \partial_{z_{i}}$, where $A=\left(a_{i j}\right)_{n \times n}$ is a constant matrix. As consequence, the holonomies have the same linear part. Hence, the map $\varphi$ has the form $(x, \mathbf{z})$ $\bmod \mathfrak{m}^{2}$, and as $\Gamma:=\{\mathbf{z}=0\}$ and $\Omega:=\{\mathbf{w}=0\}$, we have $\left.\varphi\right|_{\{\mathbf{z}=0\}}=i d$.

The restrictions of $X$ to the curves $\alpha_{x}$ and $\beta_{x}$ are equivalent to the equations below, where $\gamma_{1}$ and $\gamma_{2}$ are the respective solutions

$$
\left\{\begin{array}{l}
\frac{\partial \mathbf{z}}{\partial t}=A \mathbf{z} \quad \bmod \mathfrak{m}^{2} \\
\mathbf{z}(0)=\mathbf{z} \\
\gamma_{1}(t)=\exp (t A) \mathbf{z} \quad \bmod \mathfrak{m}^{2}
\end{array},\left\{\begin{array}{l}
\frac{\partial \mathbf{z}}{\partial t}=-2 \pi i A \mathbf{z} \quad \bmod \mathfrak{m}^{2} \\
\mathbf{z}(0)=\mathbf{z} \\
\gamma_{2}(t)=\exp (-2 \pi i t A) \mathbf{z} \quad \bmod \mathfrak{m}^{2}
\end{array}\right.\right.
$$

Then, there exists a constant $\sigma \in \mathbb{C}$ such that, the compositions of the lifts are

$$
\begin{aligned}
f_{\mathcal{F}}^{\beta_{x}} \circ f_{\mathcal{F}}^{\alpha_{x}}(x, \mathbf{z}) & =(1, \exp (\sigma A) \mathbf{z}) \quad \bmod \mathfrak{m}^{2}, \\
f_{\mathcal{G}}^{\alpha_{x}^{-1}} \circ f_{\mathcal{G}}^{\beta_{x}^{-1}}(1, \mathbf{z}) & =(x, \exp (-\sigma A) \mathbf{z}) \bmod \mathfrak{m}^{2} .
\end{aligned}
$$

We conclude that $\Psi(x, \mathbf{z})=(x, \mathbf{z}) \bmod \mathfrak{m}^{2}$ and $\Psi(x, 0)=(x, 0)$, in other words, the map $\Psi$ is an $x$-normalized automorphism.

Remark 2. Looking at the structure of the logarithm of invertible linear maps, one sees that the condition (a) of the Main Theorem can be replaced by the following non-equivalent condition: Writing the respective semi-simple parts of $X$ and $Y$ as $x \partial_{x}+L(\mu)$ and $x \partial_{x}+L(\lambda)$, no difference $\mu_{i}-\lambda_{j}, 1 \leq i, j \leq n$ is a nonzero integer.

We can reformulate the Main Theorem as follows: If the eigenvalues of $(\mathcal{F}, H, \Gamma)$ satisfy the no transverse negative resonance condition, then $(\mathcal{F}, H, \Gamma)$ is analytically classified by its linear part and its $\Gamma$-holonomy. Now, we give a proof of it.

of the Main Theorem. Let $\Psi \in \mathcal{A}_{\text {norm }}\left(\mathcal{O}_{r, R}\{\mathbf{z}\}\right)$ be the automorphism defined by Proposition 20 which conjugates $X$ to $Y$, the respective local generators, in a neighborhood of an annulus $D_{r, R}$.

By Proposition 16, there exist $\Phi_{1}, \Phi_{2} \in \mathcal{A}_{\text {norm }}\left(\mathcal{O}_{R}[[\mathbf{z}]]\right)$ which diagonalize the respective semisimple parts of $X$ and $Y$, in other words

$$
\begin{gathered}
\Phi_{1}^{*} X=X_{s}+X_{n}, \\
\Phi_{2}^{*} Y=Y_{s}+Y_{n},
\end{gathered}
$$

where $X_{s}=Y_{s}=x \partial_{x}+L(\mu)$. By the uniqueness of the Jordan decomposition, the automorphism $\Psi_{0}=\Phi_{2} \circ \Psi \circ \Phi_{1}^{-1} \in \mathcal{A}_{\text {norm }}\left(\mathcal{O}_{r, R}[[\mathbf{z}]]\right)$ is such that

$$
\Psi_{0}^{*}\left(x \partial_{x}+L(\mu)\right)=x \partial_{x}+L(\mu)
$$

It means that $\Psi_{0}$ is an $x$-normalized $D_{r, R}$-transversely formal symmetry for $x \partial_{x}+L(\mu)$. 
Let $A \circ \exp W$ be the exponential decomposition of $\Psi_{0}$ (see subsection 2.3). Applying Lemma 12, the $\exp W$ is a symmetry for $x \partial_{x}+L(\mu)$, and by Proposition 15 , we must have

$$
\left[W, x \partial_{x}+L(\mu)\right]=0 .
$$

Since $x \partial_{x}+L(\mu)$ has no transverse negative resonance, we can apply Theorem 3 to guarantee that $W \in \mathcal{D}\left(\mathcal{O}_{R}[[\mathbf{z}]]\right)$, and then, $\exp W \in \mathcal{A}\left(\mathcal{O}_{R}[[\mathbf{z}]]\right)$.

Finally, we have that $\Psi_{0}=A \circ \exp W \in \mathcal{A}\left(\mathcal{O}_{R}[[\mathbf{z}]]\right)$. As consequence, the automorphism $\Psi$ lies in the intersection $\mathcal{A}\left(\mathcal{O}_{R}[[\mathbf{z}]]\right) \cap \mathcal{A}\left(\mathcal{O}_{r, R}\{\mathbf{z}\}\right)$. Applying Lemma 4 to the components $\Psi_{1}, \ldots, \Psi_{n+1}$ of $\Psi$, we conclude that they lie in $\mathcal{O}_{R}\{\mathbf{z}\}$. Therefore, $\Psi \in \mathcal{A}_{\text {norm }}\left(\mathcal{O}_{R}\{\mathbf{z}\}\right)$.

In dimension three, we can be more precise about the local classification in terms of the linear part and the holonomy proving Corollary 2.

of Corollary 2. We recall that the triple $(1, \lambda, \mu)$ is in the Siegel (resp. Poincaré) domain if $0 \in \mathbb{C}$ belong (resp. does not belong) to the the convex hull of $1, \lambda$, and $\mu$.

If $\lambda \in \mathbb{C} \backslash \mathbb{R}$, up to a symmetry, we can assume that $\operatorname{Im}(\lambda)>0$. Then, the triple $(1, \lambda, \mu)$ is in the Siegel domain if and only if the third eigenvalue $\mu$ lies in the closed region $\mathcal{S}:=\{z \in \mathbb{C} ; \pi \leq \arg z \leq \pi+\arg \lambda\}$ (see figure 5).

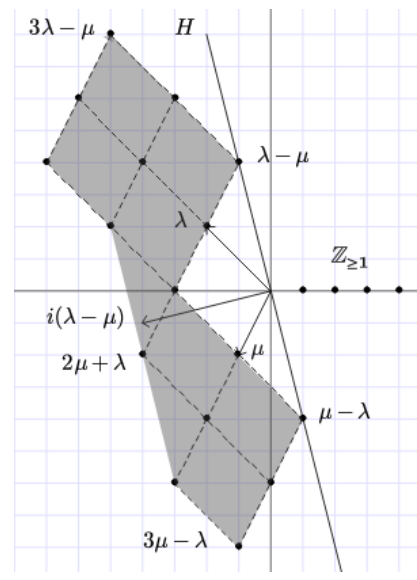

Figure 5: Siegel domain

Assume that $\mu$ lies in $\mathcal{S}$. Then, two cases can occur:

i. $\lambda$ lies in $\mathbb{C} \backslash \mathbb{R}$, and consequently the pair $(\lambda, \mu)$ satisfies the no negative resonance condition. We need to prove that the discrete positive cone

$$
\mathcal{C}=\left\{p_{1} \lambda+p_{2} \mu ; p_{1}, p_{2} \in \mathbb{Z}_{\geq-1}, p_{1}+p_{2} \geq 0\right\}
$$

contains no element $n \in \mathbb{Z}_{\geq 1}$. We consider initially the case where $\mu=0$. Then $\mathcal{C}=\left\{p_{1} \lambda ; p_{1} \in \mathbb{Z}_{\geq-1}\right\}$ and the equality $\operatorname{Im}\left(p_{1} \lambda\right)=\operatorname{Im}(n)$ implies 
that $p_{1}=0$. Consequently, we have that $0=\operatorname{Re}\left(p_{1} \lambda\right)=n$. Hence $\mathcal{C} \cap \mathbb{Z}_{\geq 1}=\varnothing$.

Now, assume that $\mu$ is nonzero. Since, $\mu \in \mathcal{S}$, the imaginary part of $\mu$ is negative. Remark that the euclidean inner product of the vectors in $\mathbb{R}^{2}$ associated to two complex numbers $z, w$ is given by $\operatorname{Re}(z \bar{w})$, and $i z$ and $-i z$ are orthogonal to $z$. Hence, the set $\mathcal{C}$ lies in the half-plane

$$
H=\{z ; \operatorname{Re}(z \overline{i(\lambda-\mu)}) \geq 0\}
$$

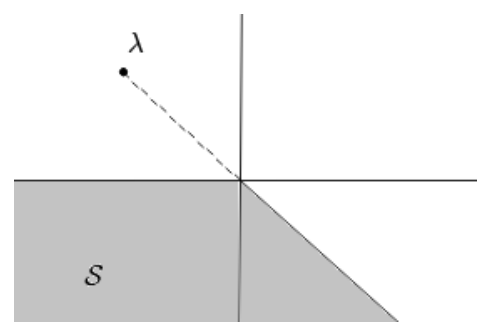

Figure 6: The discrete positive cone $\mathcal{C}$

Now, for an arbitrary positive integer number $n$,

$$
\operatorname{Re}(n \overline{i(\lambda-\mu}))=n \operatorname{Re}(\overline{i(\lambda-\mu)})=-n \operatorname{Im}(\lambda-\mu)<0
$$

which shows that $n \notin H$.

ii. $\lambda$ lies in $\mathbb{R}$, then the region $\mathcal{S}$ reduces to either to the half nonpositive real line (if $\lambda>0$ ) or to the whole complex plane (if $\lambda \leq 0$ ). We consider these two cases separately. But firstly, remark that the no transverse negative resonance property is equivalent to the conjunction of the following three conditions,

$$
\begin{array}{ccr}
\forall p_{1}, p_{2} \in \mathbb{Z}_{\geq 0}, \forall q \in \mathbb{Z}_{\geq 1} ; & p_{1} \lambda+p_{2} \mu \neq q & (\star) \\
\forall p, q \in \mathbb{Z}_{\geq 1} ; & p \lambda \neq \mu+q & (\star \star) \\
\forall p, q \in \mathbb{Z}_{\geq 1} ; & p \mu \neq \lambda+q & (\star \star \star)
\end{array}
$$

(a) $\mu \leq 0<\lambda$. Here, $p \mu \leq 0$ and $\lambda+q>0$, then the condition $(\star \star \star)$ always holds. The negation of the condition $(\star \star)$ corresponds to the equation (2), and the negation of the condition $(\star)$ is equivalent, up to a permutation of coordinates, to $p_{1} \geq 1$ and $\lambda=q / p_{1}-p_{2} / p_{1} \mu \in$ $\mathbb{Q}_{>0}-\mu \mathbb{Q}_{\geq 0}$.

(b) $\lambda \leq 0$. The case where $\mu \neq \mathbb{R}$ is treated in (i.). By a changing coordinates, the case where $\mu>0$ is the item (ii.a). Hence, up to a permutation of coordinates, just $\mu \leq \lambda \leq 0$ remains to be considered. In this case, the conditions $(\star)$ and $(\star \star \star)$ always hold. The negation of the condition ( $\star \star)$ implies that $\mu<\lambda \leq 0$ and that (2) holds. 


\section{References}

[CN77] César Camacho and Alcides Lins Neto. Introdução à Teoria das Folheações. IMPA, Rio de Janeiro, 1977.

[CN85] César Camacho and Alcides Lins Neto. Geometric theory of foliations. BIRKHAUSER, 1985.

[Dia19] Adjaratou Arame Diaw. Géométrie de certains tissus holomorphes singuliers en dimension 2. PhD thesis, Rennes 1, 2019.

[DL20] Adjaratou Arame Diaw and Frank Loray. Pairs of foliations and matteimoussu's theorem. arXiv preprint arXiv:2008.00719, 2020.

[EI84] PM Elizarov and Yu S Il'yashenko. Remarks on the orbital analytic classification of germs of vector fields. Mathematics of the USSRSbornik, 49(1):111, 1984.

[IY08] Yulij Ilyashenko and Sergei Yakovenko. Lectures on analytic differential equations, volume 86. American Mathematical Soc., 2008.

[Mar81] Jean Martinet. Normalisation des champs de vecteurs holomorphes. In Séminaire Bourbaki : vol. 1980/81, exposés 561-578, number 23 in Séminaire Bourbaki, pages 55-70. Springer-Verlag, 1981. talk:564.

[MM80] Jean-François Mattei and Robert Moussu. Holonomie et intégrales premières. Annales scientifiques de l'École Normale Supérieure, 4e série, 13(4):469-523, 1980.

[MR82] Jean Martinet and Jean-Pierre Ramis. Problemes de modules pour des équations différentielles non linéaires du premier ordre. Publications Mathématiques de l'Institut des Hautes Études Scientifiques, 55(1):63164, 1982.

[Rei06] Helena Reis. Equivalence and semi-completude of foliations. Nonlinear Analysis: Theory, Methods \& Applications, 64(8):1654-1665, 2006.

[Ser09] Jean-Pierre Serre. Lie algebras and Lie groups: 1964 lectures given at Harvard University. Springer, 2009. 